\title{
PROMISING EFFECT OF DAPAGLIFLOZIN ON OVARIECTOMIZED RAT MODEL OF OSTEOPOROSIS
}

\section{Amany Ali Nawar ${ }^{1}$, Nancy Mohamed El Sekily *2.}

${ }^{1}$ Clinical pharmacology Department, Faculty of Medicine, Alexandria University, Egypt.

${ }^{* 2}$ Anatomy and Embryology Department, Faculty of Medicine, Alexandria University, Egypt.

\section{ABSTRACT}

Introduction: Osteoporosis makes bones become frail thus fragile, that a fall or even gentle burdens prompting a crack. Several studies had documented that osteoporosis incidence is increased much more in diabetic patient. Dapagliflozin, is used in Type 2 diabetes management, as a selective inhibitor of sodium/glucose cotransporter2 (SGLT2). Few study tackled the impact of dapagliflozin on bone metabolism. In this study we aim to determine the impact of dapagliflozin on a rat model of osteoporosis.

Materials and methods: Thirty adult female Wistar albino rats were used in this experiment. They were equally divided into three groups. Group I sham-operated control, group II ovariectomized (OVX), and group III ovariectomized (OVX) treated with dapagliflozin (DAPA) $10 \mathrm{mg} / \mathrm{kg} /$ day orally for 8 weeks. After 8 weeks, plasma levels of calcium $(\mathrm{Ca}+2)$, phosphorous (P), alkaline phosphatase (ALP), Tartrate-Resistant Acid Phosphatase $5 \mathrm{~b}$ (TRAP 5b), tumor necrosis factor- $\alpha$ (TNF- $\alpha$ ), serum osteocalcin level, serum osteoprotegerin level, AMP-activated protein kinase (AMPK) and advanced glycosylation end products (AGEs) were analysed. The distal end of femur bones were excised for histological and morphometric studies.

Results: In the current study, the biochemical results of ovariectomized (OVX) rat group showed increase in serum level of phosphorus, alkaline phosphatase, Tartrate-Resistant Acid Phosphatase 5b (TRAP 5b), osteocalcin, advanced glycosylation end products (AGEs) and tumor necrosis factor- $\alpha$ (TNF- $\alpha$ ). While the serum level of calcium, osteoprotegrin and AMP-activated protein kinase (AMPK) was decreased. The biochemical results of (OVX) group treated with DAPA showed a significant change in serum level of calcium, alkaline phosphatase, TRAP 5b, osteocalcin, osteoprotegrin and AMPK. While the serum level of phosphorus, AGEs and TNF- $\alpha$ showed non significant change in the same group. In the present study, the (OVX) rat group showed features of osteoporosis at the epiphyseal plate of femur. These features were focal areas of disrupted arrangement of the layers of chondrocytes, some areas of the matrix appeared pale, the bone trabeculae were thinner than normal, multiple resorption cavities and some areas of hypocellularity. After oral administration of dapagliflozin to the OVX rats for eight weeks, the osteoporotic findings in femur by H\&E showed improvement. The chondrocytes appeared almost normally organized and the bone trabeculae were of normal thickness. By morphometric assessment, the thickness of bone trabeculae of OVX treated with DAPA group (69.9 \pm 15.6$)$ increased in comparaison to the OVX group (61.0 18.2$)$.

Conclusion: Dapaglaflozin has an antiosteoporotic effect in ovariectomy induced osteoporosis in rat.

KEY WORDS: Dapagliflozin, Osteoporosis, bilateral ovaricteomy.

Corresponding Author: Nancy Mohamed Aly El-Sekily, Anatomy and Embryology, Department, Faculty of Medicine, Alexandria University, Egypt. +0201222359100

E-Mail: nancyelsekily@yahoo.com

Access this Article online

Quick Response code

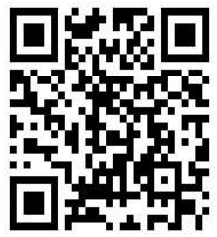

DOI: $10.16965 /$ ijar.2020.204
Journal Information

International Journal of Anatomy and Research

RG Journal ISSN (E) 2321-4287 | ISSN (P) 2321-8967

Impact: 0.21* https://www.ijmhr.org/ijar.htm

DOI-Prefix: https://dx.doi.org/10.16965/ijar

Article Information

Received: 09 Aug 2020

Peer Review: 09 Aug 2020

Revised: None
Accepted: 20 Aug 2020

Published (O): 10 Sep 2020

Published (P): 10 Sep 2020 


\section{INTRODUCTION}

Osteoporosis makes bones become frail thus fragile, that a fall or even gentle burdens prompting a crack. These osteoporosis-related fractures commonly occur in hip, wrist or vertebrae [1].

It is notable that females of all races are in danger for osteoporosis but post-menopausal females are at higher risk. Several studies had documented that osteoporosis incidence is increased much more in diabetic patient [2]. These could be whether due to the disease itself or the antidiabetic drugs as pioglitazones. There is growing evidence that increased glucose level in diabetes mellitus (DM) and accumulation of the advanced glycosylation end products (AGEs) in the collagen fibers allowed alteration of bone structure and strength. Moreover, alteration in plasma insulin level and alteration and disturbance in renal function also affects bone microstructure as it decreases vascular flow leading to bone microangiopathy. In addition chronic inflammation and increased of cytokines levels had an implemented role in accelerating bone remodeling and loss of bone mineral density (BMD) [3].

Dapagliflozin, is used for treatment of Type 2 diabetes, as a selective inhibitor of sodium/ glucose cotransporter-2 (SGLT2) [4]. There is growing evidence that presence of DM builds the danger of secondary osteoporosis and fracture irrespective to diabetic clinical type. A lot of studies implicated the roles of AGEs in aggravating both bone fragility, as well as progression of classical diabetic complications $[5,6]$.

AGEs are closely associated with the deterioration of the material properties of bone. As AGEs significantly affects both osteoblast and osteoclast, it inhibits osteoblast proliferation and induces its premature apoptosis, [7] besides it suppresses osteoblastic differentiation [8]. AGEs diminished osteoclast-prompted bone resorption and the osteoclastic separation [9]. AGEs increased the expression of the antagonist of bone formation; sclerostin protein, and diminished the expression of the agonist of bone resorption; RANKL protein [10].

AMPK signaling pathway plays a role in bone physiology. Novel work demonstrates that several pathways influencing bone formation could be modulated through activation of AMPK, as mevalonate pathway, AMPK modulates it by repression of HMG-CoA reductase [11] stimulating osteoblast differentiation, osteoclastic bone resorption and bone development [12].

Also there is increasing evidence that guideline of osteoblast separation through crosstalk with the Wnt/b-catenin pathway that could be modulated by activation of the AMPK [13].

Furthermore, activation of the AMPK pathway stimulates osteoblast differentiation and bone formation via enhancing the osteoblast-specific transcription factor (runt-related transcription factors 2) RUNX2 and also inhibition of osteoclast formation and bone resorption possibly via suppression of the nuclear factor of activated $T$ cells c1 (NFATc1) [14].

Few study tackled the impact of dapagliflozin on bone metabolism, and these studies specifically study its effect on diabetic patients where several variables as DM itself and metformin combination drugs are present. In this study we aim to determine effect of dapagliflozin on a rat model of osteoporosis.

\section{MATERIALS AND METHODS}

Chemicals: Dapagliflozin (Oral tablets: $10 \mathrm{mg}$ ) was purchased from Bristol-Myers Squibb (American pharmaceutical company). The drug was dissolved in saline as a vehicle.

Animals: Thirty adult female Wistar albino rats weighing from $150-200 \mathrm{~g}$ at age between $10-24$ months with regular estrous cycles were picked as suggested by Kalu [15]. The rats were obtained from the animal house of department of Anatomy and Embryology, Faculty of Medicine, Al - Mowasat. The animals were housed on wood shavings in metal cages and were kept in animal house, Faculty of medicine, Alexandria University under standard conditions of light and temperature with free access to food and water. The current study was affirmed by the Ethical rules of Alexandria University on laboratory animals and the national institute for the care and use of laboratory animals (NHI Publications No80-23, revised 1978). Number of the ethical approval: 0304099 and date of the ethical approval: 20/09/2018. Further the 
Alexandria faculty of medicine ethical committee approval was acquired.

In the experiment, female albino rats were equally divided into three groups:

Group I sham-operated control (SHAM): included ten female adult rats that were given $1 \mathrm{ml}$ saline every day orally for 8 weeks.

Group II ovariectomized (OVX) [15,6]: Included ten female adult rats that undergo bilateral ovaricteomy using dorsal approach under general anesthesia. The rats were given $1 \mathrm{ml}$ saline every day orally for 8 weeks.

Group III ovariectomized (OVX) treated with DAPA: included ten female adult rats that were ovariectomized treated with dapagliflozin 10 $\mathrm{mg} / \mathrm{kg} /$ day [16] dissolved in $1 \mathrm{ml}$ saline every day orally for 8 weeks.

After 8 weeks, the rats were anaesthetized by ether then blood was collected from the aorta to analyse plasma levels of calcium $(\mathrm{Ca}+2)$, phosphorous (P), alkaline phosphatase (ALP), tumor necrosis factor- $\alpha$ (TNF- $\alpha$ ), serum osteocalcin level, serum osteoprotegerin level, AMP-activated protein kinase (AMPK), advanced glycosylation end products (AGEs) and the distal end of femur bones were excised for histological and morphometric studies.

Ovariectomy [17]: Animals were anaesthetized with ketamine $(100 \mathrm{mg} / \mathrm{kg})$ via an intraperitoneal route [18] and injected intramuscularly with antibiotics (penicillin $\mathrm{G}$ procaine, $40.000 \mathrm{U} / \mathrm{kg}$ ). A single transverse incision was made in the ventral midline, through the skin and the muscle anterior abdominal wall at the lower poles of the kidneys. The two ovaries were then located and silk threads (5-0) were tightly tied around the oviducts, including the ovarian blood vessels. The oviducts were sectioned, and the ovaries were removed with their surrounding fat, taking good care in leaving the knots intact. The skin and the muscle wall were then sutured with a silk thread (4-0).

- All administered drugs or vehicle were given by an oral gavage syringe.

- All administered drugs were dissolved in $1 \mathrm{ml}$ saline.

Biochemical laboratory estimates:

Calcium and phosphorus assay: Calcium and phosphorus levels were estimated in serum using colorimetric assay kits one for calcium and another for phosphorus (LINEAR CHEMICALS S.L., Barcelona, Spain), according to the manufacture's instruction.

Alkaline phosphatase assay: The alkaline phosphatase activity was quantified in serum using ALP colorimetric assay kit (BIODIAGNOSTIC, Egypt), according to the manufacture's instruction.

Tartrate-Resistant Acid Phosphatase 5b (TRAP

5b) assay: Serum TRAP 5b concentrations were quantified using a Rat ELISA kit, purchased from WKEA MED SUPPLIES CORP, China according to the manufacture's instruction.

Osteocalcin and Osteoprotegerin assay:Serum levels were quantified using rat osteocalcin ELISA kit, and rat Osteoprotegerin ELISA kit purchased from (Cloud-Clone Corp-USCN life science inc. - USA), according to the manufacture's instruction.

AMP-activated protein kinase (AMPK): Serum AMPK was measured by Rat AMPK ELISA kit (MyBiosource, Inc. San Diego. USA), according to the manufacture's instruction.

Advanced glycosylation end products (AGEs): Serum AGEs was measured by Rat AGEs ELISA kit (MyBiosource, Inc. San Diego. USA), according to the manufacture's instruction.

Tumor necrosis factor- $\alpha$ (TNF- $\alpha$ ) assay: Serum TNF- $\alpha$ was measured by the RayBio ${ }^{\circledR}$ Rat TNF- alpha ELISA kit (RayBiotech, Inc., Norcross, Georgia, USA), according to the manufacture's instruction.

Histological and morphometric studies: Distal end of femur bones were excised using bone cutter and cleaned for any remains of muscle fibers. The bones were fixed in $10 \%$ formalaldehyde, decalcified using the chelating agent ethylene diamine tetra acetic acid (EDTA) then placed in molds and embedded in paraffin to form paraffin blocks for histomorphometric parameters. The distal end of femur bones were sectioned (3-5um thickness) longitudinally and processed for Hematoxylin and Eosin ( $\mathrm{H}$ and $\mathrm{E}$ ) staining [19] and Gomori's trichrome stain [20]. Digital images from H\&E stained sections were obtained by digital camera connected to microscope (Olympus BX41). The magnification of 
images were $(100 \mathrm{X})$ and $(400 \mathrm{X})$. The bone trabecular thickness using images at $100 \mathrm{X}$ magnification. Measurements were expressed as length $(\mu \mathrm{m})$ using NIH Image J (v1.49) software.

Statistical Analysis: The results of this study were interpreted as mean \pm standard deviation (SD). Statistical analyses were performed with IBM SPSS statistics, version 23.0(IBM Inc). All experimental results were analyzed utilizing one-way variance analysis (ANOVA) with Dunnett's post hoc test. Values of $P \leq 0.05$ were were significantly different.

\section{RESULTS}

There was no mortality observed during the experimental period.

\section{Biochemical results:}

The effect of eight weeks treatment with Dapagliflozin on serum calcium, phosphorus (mg/dl), alkaline phosphatase (IU/L) and Tartrate-Resistant Acid Phosphatase 5b (TRAP $5 b)(\mathrm{pg} / \mathrm{L})$ levels in ovariectomy-induced osteoporosis in rats Fig. 1(a-d)

- Osteoporosis induction by ovariectomy (OVX group) resulted in a statistically significant decrease in serum $\mathrm{Ca}$ level and significant increase in serum $P(P<0.05)$ when compared to the normal control group.

- Eight weeks oral treatment with Dapagliflozin in ovariectomized rats were associated with a significant change in serum Ca level compared to the OVX control group $(P<0.05)$ but non- significant change in serum $P$ to the $O V X$ control group.

- Osteoporosis induction by ovariectomy (OVX group) resulted in a statistically significant increase in serum alkaline phosphatase level $(P<0.05)$ when compared to the normal control group.

- Eight weeks oral treatment with Dapagliflozin in ovariectomized rats were associated with a significant change in serum alkaline phosphatase level compared to the OVX control group $(P<0.05)$ but non-significant change compared to normal group.

- Osteoporosis induction by ovariectomy (OVX group) resulted in a statistically significant increase in serum TRAP $5 b$ level $(P<0.05)$ when compared to the normal control group.

Eight weeks oral treatment with Dapagliflazone in ovariectomized rats were associated with a significant change in serum TRAP $5 \mathrm{~b}$ level compared to the OVX control group $(P<0.05)$ but non-significant change compared to normal group.
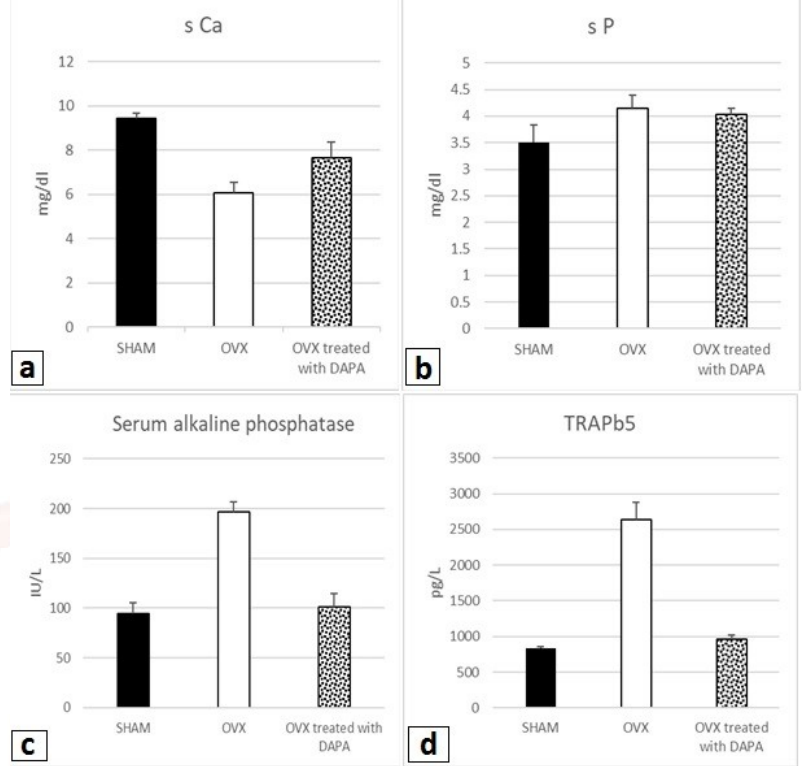

Fig. 1(a-d): Bar chart showing the mean of serum Ca, $P$, alkaline phosphatase and TRAP $5 \mathrm{~b}$ level among the different study groups.

The effect of eight weeks treatment with Dapagliflozin on osteocalcin level $(\mathrm{ng} / \mathrm{ml})$ in ovariectomy- induced osteoporosis in rats (Fig. 2)

- Osteoporosis induction by ovariectomy (OVX group) resulted in a statistically significant increase in serum osteocalcin level $(P<0.05)$ when compared to the normal control group.

- Eight weeks oral treatment with Dapagliflozin in ovariectomized rats were associated with a significant change in serum osteocalcin level compared to the OVX control group $(P<0.05)$ but non-significant change compared to normal group.

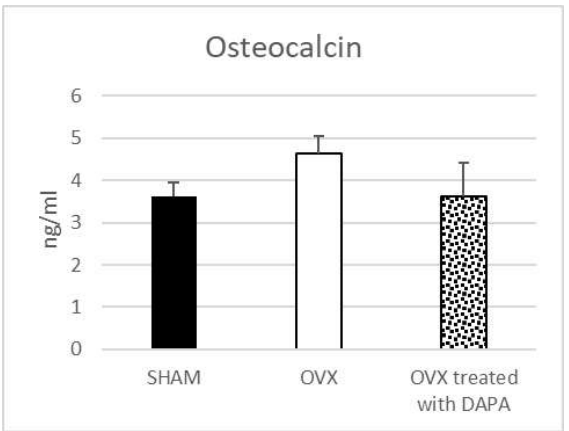

Fig. 2: Bar chart showing the mean of serum osteocalcin among the different study groups. 
The effect of eight weeks treatment with Dapagliflozin on osteoprotegrin $(\mathrm{ng} / \mathrm{ml})$, AMPK (ng/ml), AGEs (ng/ml), and TNF $\alpha(\mathrm{pg} /$ $\mathrm{ml}$ ) levels in ovariectomy- induced osteoporosis in rats Fig. 3(a-d)

- Osteoporosis induction by ovariectomy (OVX group) resulted in a statistically significant decrease in serum osteoprotegrin and AMPK levels and significant increase in serum AGEs, and TNF $\alpha$ level $(P<0.05)$ when compared to the normal control group.

- Eight weeks oral treatment with Dapagliflozin in ovariectomized rats were associated with a significant increase in serum osteoprotegrin and AMPK levels and significant decrease in serum AGEs, and TNF $\alpha$ levels compared to the OVX control group $(P<0.05)$.

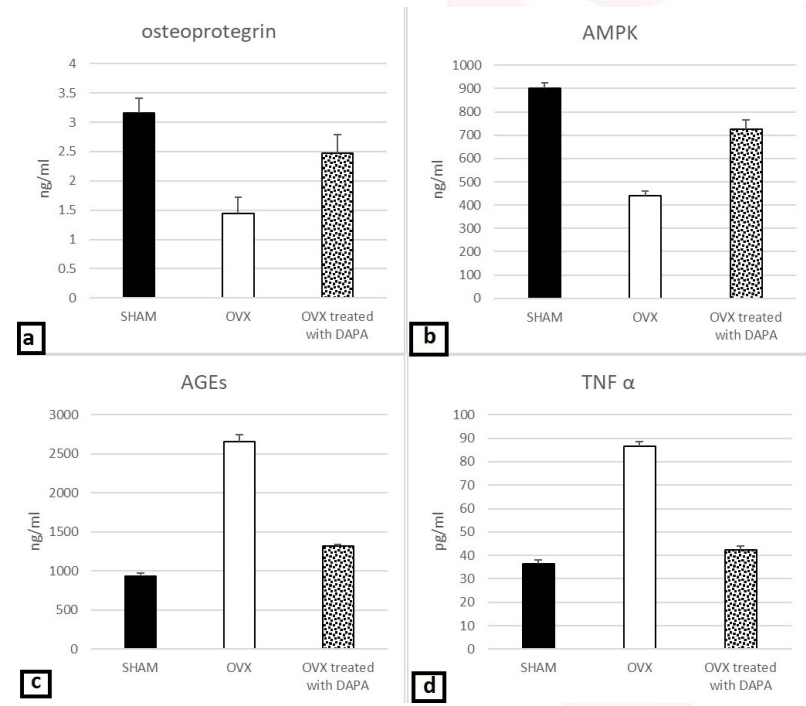

Fig. 3(a-d): Bar chart showing the mean of serum osteoprotegrin, AMPK, AGEs and TNF- $\alpha$ level among the different study groups.

Histological and morphometric assessment of distal end of femur;

Observations of H\&E stained sections: By light microscope examination of the control group of female albino rats, normal histological features of cancellous bone at the epiphyseal plate of femur had been showed (Fig.4a). The epiphyseal plate of femur of (OVX) rat showed features of osteoporosis. Focal areas of disrupted arrangement of the layers of chondrocytes and some areas of the matrix appeared pale. The bone trabeculae were thinner than normal (Fig.4b). Multiple resorption cavities also appeared within the bone (Figs. 4c, d). Some areas of hypocellularity was also seen (Fig.4d). Morphometric analysis of (OVX) group revealed that bone trabecular thickness $(61.0 \pm 18.2)$ was significantly decreased in comparison to the control group (76.1 \pm 13.1 ).

The (OVX) rat who received DAPA orally for 8 weeks showed improvement of osteoporosis features. The chondrocytes appeared almost normally organized; the bone trabeculae were of normal thickness (Fig. 4e).

Observations of Gomori's Trichrome stained sections: Gomori's Trichrome stained sections of (OVX) group (Fig. 5b ) showed few collagen fibers in the trabecular bone that appeared widely separated by bone marrow rich in fat cells compared to control group (Fig. 5 a). The (OVX) rat who received DAPA orally revealed nearly normal content and distribution of collagen fibers in the trabecular bone which indicates improvement of osteoporotic features (Fig. 5C).

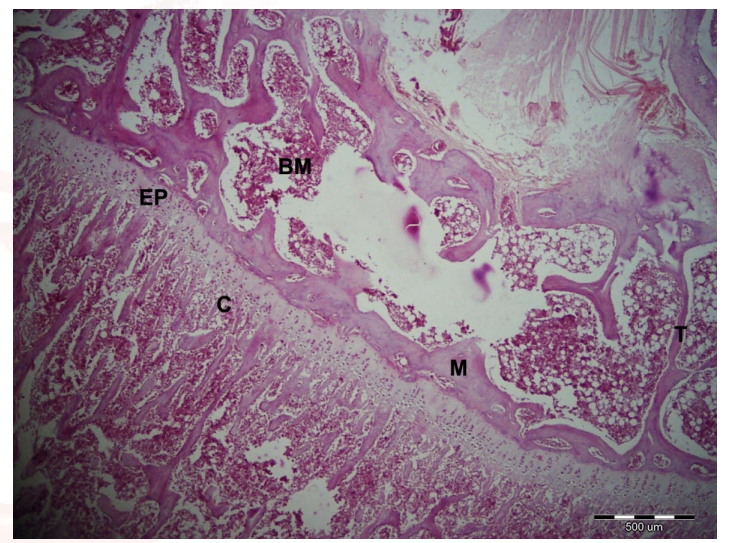

Fig. (4a): Photomicrograph of the sham-operated control group showing normal histological appearance of epiphyseal plate of hyaline cartilage. Normal appearance of cancellous bone $(\mathrm{C})$ in the epiphysis. The cartilage matrix (M) appears homogenously stained. Bone marrow cavities appear (BM) T: trabeculae. (H\&E stain, Mic.Mag. x 100)

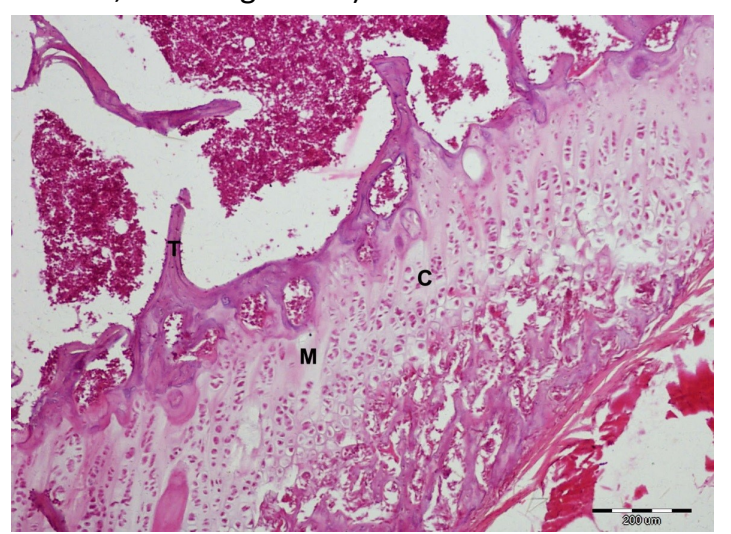

Fig. (4b): Photomicrograph of (OVX) group showing focal areas of disrupted arrangement of the layers of chondrocytes (C) and disrupted arrangement of cancellous bone at the epiphyseal plate. Some areas of the matrix (M) appear pale. Bone trabeculae $(T)$ appear thinner than normal. (H\&E stain, Mic.Mag. x 100) 


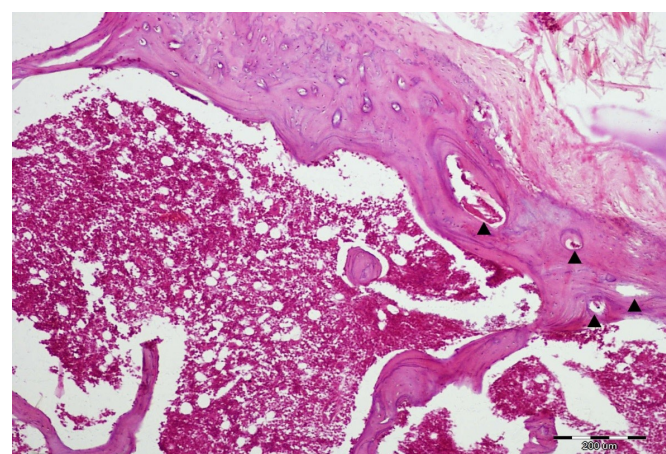

Fig. (4c): Photomicrograph of ovariectomized group showing the bone with multiple resorption cavities $(\mathbf{\Delta})$. (H\&E stain, Mic.Mag. x 400)

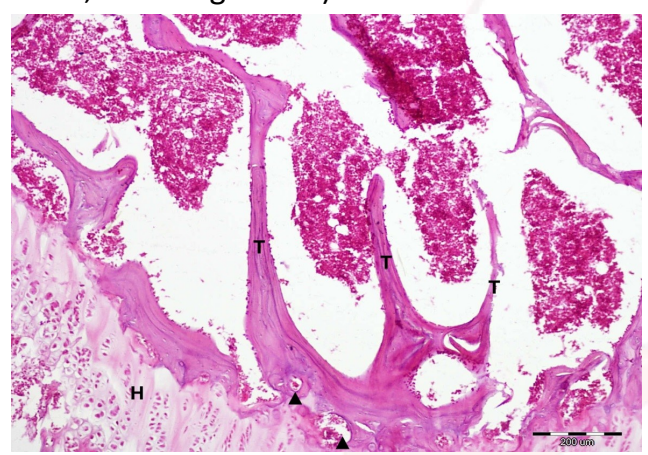

Fig. (4d): Photomicrograph of (OVX) group showing thinning of bone trabeculae $(T)$ with appearance of multiple resorption cavities ( $\boldsymbol{\Delta}$ ). Some areas of hypocellularity appear (H). (H\&E stain, Mic.Mag. x 100)

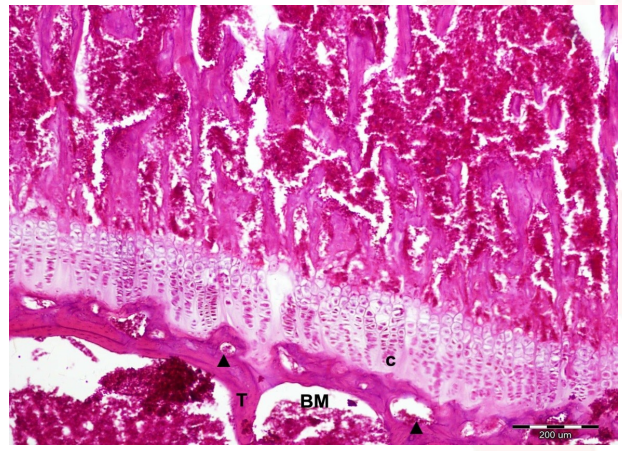

Fig. (4 e): Photomicrograph of (OVX) group treated with DAPA showing improvement of histological features, almost normally organized chondrocytes (C) and decreased resorption cavities(R). Bone marrow cavities (BM) appear separated by bone trabeculae (T). (H\&E stain, Mic.Mag. x 100)

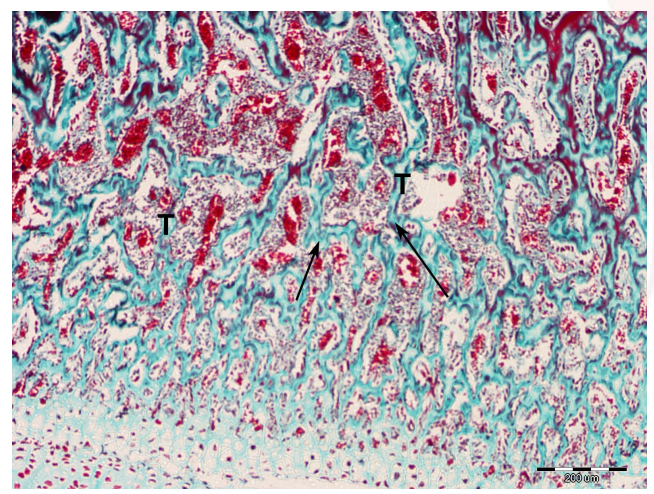

Fig. (5 a): Photomicrograph of the sham-operated control group showing normal distribution of collagen fibers ( $\uparrow$ ) in bone trabeculae (T).(Gomori's Trichrome stain, Mic.Mag X 100)

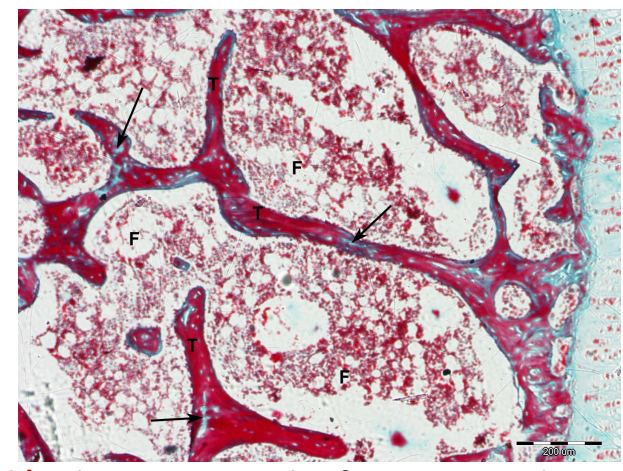

Fig. (5b): Photomicrograph of OVX group showing few collagen fibers ( $\uparrow$ ) in the trabecular bone $(T)$ that appeared widely separated by bone marrow rich in fat cells (F).(Gomori's Trichrome stain, Mic.Mag X 100)

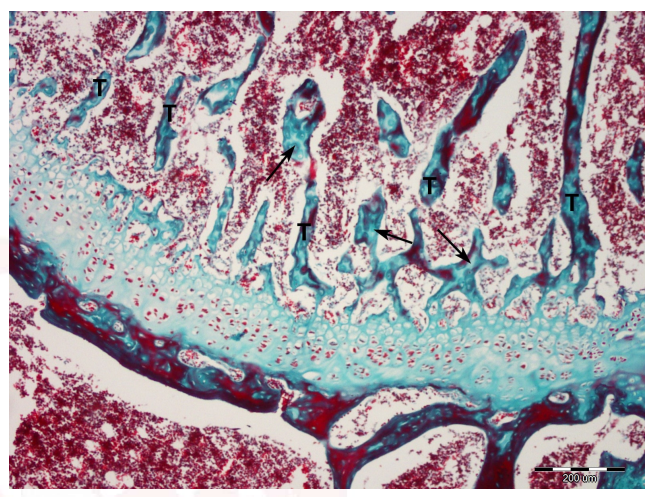

Fig. (5c): Photomicrograph of (OVX) group treated with DAPA showing nearly normal content and distribution of collagen fibers $(\uparrow)$ in the trabecular bone $(T)$. (Gomori's Trichrome stain, Mic.Mag X 100)

Table (1) shows increase in the trabecular thickness of ovariectomized treated with DAPA group when compared with control group. Then again, there was a decrease in the trabecular thickness of (OVX) group in comparaison to the control group (Figure 6).

The results of the study in (Figure 6) show that the average trabecular thickness in the control, OXV and OXV treated with DAPA groups were respectively 76.1; 61.0; and $69.9 \%$. The mean trabecular thickness was highest in control group and lowest in OXV group.

Table 1: Comparison between the three groups regarding trabecular thickness.

\begin{tabular}{cccc}
\hline Percent of area & Control & OVX & $\begin{array}{c}\text { OVX treated } \\
\text { with DAPA }\end{array}$ \\
\hline Range & $47.8-89.9$ & $25.3-83.4$ & $49.5-88.9$ \\
Mean & 76.1 & 61 & 69.9 \\
S.D. & 13.1 & 18.2 & 15.6 \\
\hline ANOVA & & 12.11 & \\
P & & $0.021^{*}$ & \\
\hline P1 & & $0.023^{*}$ \\
P2 & & $0.011^{*}$ \\
P3 & & $0.047^{*}$ \\
\hline
\end{tabular}

$P$ is significant if $\leq 0.05$; $P 1$ comparison between control and (OVX) treated with DAPA; P2 comparison between control and (OVX); $\mathrm{P} 3$ comparison between (OVX) treated with DAPA and (OVX) 


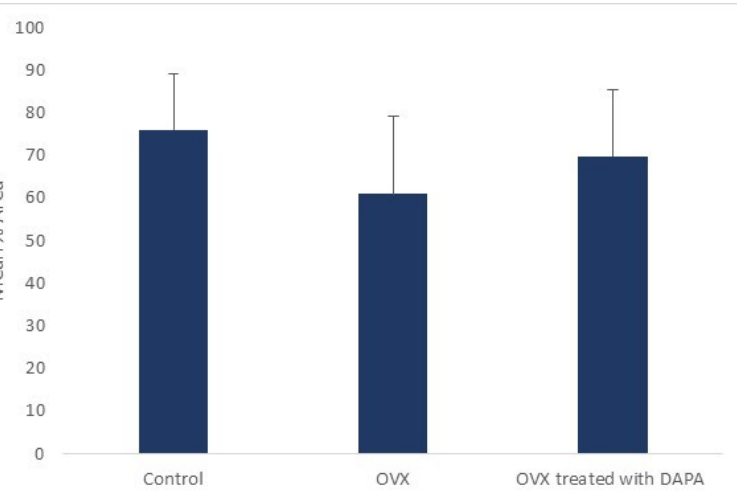

Fig. (6): Bar chart showing mean $\%$ area among studied groups.

\section{DISCUSSION}

Osteoporosis is a metabolic bone disease where there is both a decrease in the ordinary amount of mineralized bone as an adjustment in bone microarchitecture, prompting expanded break hazard in specific locales, even without high-vitality injury. It affects $25 \%$ of women over 60 years old and $5 \%$ to $10 \%$ of men in the same age group in western societies [21].

The (OVX) rat model remains the most famous choice as it has been approved to represent the most significant clinical highlights of estrogen deficiency-induced (or postmenopausal) bone loss in the adult human [22] particularly during the beginning phases of osteoporosis. These include: increased rate of bone turnover with resorption surpassing arrangement; an underlying fast period of bone loss followed by a much more slow stage; more loss of cancellous bone than cortical bone [23].

Dapagliflozin, is a highly selective inhibitor of sodium-glucose cotransporter 2 (SGLT2) the newest class of oral anti-diabetics [24]. It improves glycaemic control in patients with type 2 diabetes mellitus (T2DM) when used as monotherapy by increasing urinary glucose excretion [25].

Current evidence suggests that Tumor necrosis factor-alpha (TNF- $\alpha$ ) promotes bone remodeling and osteoporosis by enhancing osteoclast formation and depressing osteoblast maturation and function. This is through its role in chronic inflammation and enhancement of receptor activator of nuclear factor kappa ß (RANK) and osteoclast precursor cell proliferation [26].

AMPK is a key target in the pathophysiology of osteoporosis; it is enrolled in many pathways impacting osteoporosis.

Relevant findings in the current study revealed that administration of dapagliflozin to ovarectomized female rat induced osteoporosis improved the histological, morphometric studies and biochemical parameters.

Dapagliflozin treated ovariectomy- induced group significantly increases serum calcium, osteoprotegrin, AMPK, and significantly decreases serum alkaline phosphatase level, serum Tartrate-Resistant Acid Phosphatase $5 b$ (TRAP 5b), osteocalcin level, AGEs, and TNF $\boldsymbol{\alpha}$ levels compared to ovariectomy-induced osteoporosis group.

Effect of Dapagliflozin as an activator to AMPK was in line with some studies [27, 28, 29]. Also, Yao $D$ et al, approved antioxidant and antiinflammatory effect of Dapagliflazone [30, 31]. On the other hand, some studies documented that dapagliflozin modulated SRAGE levels, suggesting that dapagliflozin had inhibitory effect on AGE-RAGE axis [32, 33].

The present study revealed that administration of dapagliflozin led to a significant decrease in the AGEs level. This finding illustrates the importance of the dapagliflozin use of in diabetic especially postmenopausal and osteoporotic patient.

Some clinical studies suggest that Dapagliflozin had no effect on bone formation markers and resorption or bone mineral density in diabetic type 2 patients. These could be explained that osteoporotic changes had been established in bone of these patients, before starting treatment of dapagliflozin [34, 35].

Fundamental clinical information have appeared no changes in serum calcium or vitamin $D$ but small increases in serum magnesium, phosphate and parathyroid hormone with dapagliflozin treatment compared with placebo $[36,37]$.

During dapagliflozin treatment, serum phosphate levels increased by $9 \%$ compared to placebo [38].

In the current study, the (OVX) rat group showed features of osteoporosis at the epiphyseal plate of femur. These features were focal areas of disrupted arrangement of the layers of chondrocytes, some areas of the matrix appeared 
pale, the bone trabeculae were thinner than normal, multiple resorption cavities and some areas of hypocellularity. These results were in accordance with results of Hafiza A. S et al. [39] which revealed many osteoporotic cavities, resorption cavities, and calcified cartilage in bone tissue in the case of OVX rats. In their study, examination of cancellous bone in the head of the femur revealed decreased mean areas of bone trabeculae $(276.08 \mu \mathrm{m} 2$ ) as compared with the sham-operated group $(1220.9 \mu \mathrm{m} 2)$. In the present study the mean thickness of bone trabeculae in OVX rats $(61.0 \pm 18.2)$ was decreased as compared to the control group (76.1 \pm 13.1). Smith $R,[40]$ declared that histological assessment of osteoporotic bone showed generalized thinning of trabeculae and reflecting unequal osteoclast-mediated bone resorption. Both studies were in agreement with the findings of the present study.

In the current study, the osteoporotic findings in femur by H\&E showed improvement in OVX treated with DAPA group. The chondrocytes appeared almost normally organized and the bone trabeculae were of normal thickness. By morphometric assessment, the thickness of bone trabeculae of OVX treated with DAPA group (69.9 \pm 15.6$)$ increased in comparison to the OVX group $(61.0 \pm 18.2)$. These results were in accordance with Wang et al. [41] who recommended in their observations that Dapagliflozin has protective impacts on bone in Type 2DM people. In contrast, Kohan et al. [42] reported fractures at sites for example the toes and patellae, which may be more probable identified with falls, with dapagliflozin treatment in patients with respectably impeded renal capacity. The review noted in one trial that $10 \mathrm{mg}$ dapagliflozin experience more fractures contrasted with lower doses ( 2.5 and $5 \mathrm{mg} /$ day) (9.4\% (8/85) of patients getting $10 \mathrm{mg} / \mathrm{day}$ dapagliflozin and $6.0 \%(5 / 83)$ of patients treated with $5 \mathrm{mg} /$ day dapagliflozin more than 104 weeks of follow-up, though no fractures were accounted for in patients receiving placebo.

\section{CONCLUSION}

Dapaglaflozin has an antiosteoporotic effect in ovariectomy induced osteoporosis in rat.

Funding: This study has no special funding from any organization.

Ethical approval: The current study was approved by the Ethical guidelines of Alexandria University on laboratory animals and the national institute for the care and use of laboratory animals (NHI Publications No80-23, revised 1978). Further the Alexandria Faculty of Medicine ethical committee approval was obtained.

Conflict of Interest: The authors proclaim that there is no conflict of interest regarding the publication of this paper.

\section{REFERENCES}

[1]. Shuid AN, Ping LL, Muhammad N, Mohamed N, Soelaiman IN. The effects of Labisia pumila var.alata on bone markers and bone calcium in a rat model of post-menopausal osteoporosis. J Ethnopharmacol 2011;133(2):538-542.

https://doi.org/10.1016/j.jep.2010.10.033 PMid:20971181

[2]. Sambrook PN, Eisman JA. Osteoporosis prevention and treatment. Med J Aust. 2000;172(5):226-9. h t t p s://doi.org/10.5694/j.13265377.2000.tb123917.x PMid:10776395

[3]. Russo GT, Giandalia A, Romeo EL, Nunziata M, Muscianisi M, Ruffo MC, Catalano A, Cucinotta D. Fracture Risk in Type 2 Diabetes: Current Perspectives and Gender Differences. Int J Endocrinol. 2016;1615735.

https://doi.org/10.1155/2016/1615735

PMid:28044077 PMCid:PMC5164892

[4]. Yang DH, Chiang TI, Chang IC, Lin FH, Wei CC, Cheng YW. Increased levels of circulating advanced glycation end-products in menopausal women with osteoporosis. Int J Med Sci. 2014;11(5):453-60. https://doi.org/10.7150/ijms.8172 PMid:24688308 PMCid:PMC3970097

[5]. Goh SY, Cooper ME. Clinical review: the role of advanced glycation end products in progression and complications of diabetes. J Clin Endocrinol Metab. 2008;93(4):1143-52.

https://doi.org/10.1210/jc.2007-1817 PMid:18182449

[6]. Masahiro Yamamoto1 \& Toshitsugu Sugimoto. Advanced Glycation End Products, Diabetes, and Bone Strength. Curr Osteoporos Rep. 2016;14:320-326. https://doi.org/10.1007/s11914-016-0332-1 PMid:27704396 PMCid:PMC5104767

[7]. AlikhaniM, Alikhani Z, Boyd C, MacLellan CM, Raptis $M$, Liu $R$, et al. Advanced glycation end products stimulate osteoblast apoptosis via the MAP kinase and cytosolic apoptotic pathways. Bone. 2007;40(2):345-53. https://doi.org/10.1016/j.bone.2006.09.011 PMid:17064973 PMCid:PMC1913208 
[8]. Ogawa N, Yamaguchi T, Yano S, Yamauchi M, Yamamoto M, Sugimoto T. The combination of high glucose and advanced glycation end-products (AGEs) inhibits the mineralization of osteoblastic MC3T3-E1 cells through glucose-induced increase in the receptor for AGEs. Horm Metab Res. 2007;39(12):871-5.

https://doi.org/10.1055/s-2007-991157 PMid:17960513

[9]. Valcourt U, Merle B, Gineyts E, Viguet-Carrin S, Delmas PD, Garnero P. Non-enzymatic glycation of bone collagen modifies osteoclastic activity and differentiation. J Biol Chem. 2007;282(8):5691-703. https://doi.org/10.1074/jbc.M610536200 PMid:17142454

[10]. Tanaka K, Yamaguchi T, Kanazawa I, Sugimoto T. Effects of high glucose and advanced glycation end products on the expressions of sclerostin and RANKL as well as apoptosis in osteocyte-like MLOY4- A2 cells. Biochem Biophys Res Commun. 2015; 461 ( 2):193-9.https://doi.org/10.1016/ j.bbrc.2015.02.091 PMid:25721666

[11]. Oliaro-Bosso S, Calcio Gaudino E, Mantegna S, Giraudo E, Meda C, Viola F \& Cravotto G. Regulation of $\mathrm{HMGCOA}$ reductase activity by policosanol and octacosadienol, a new synthetic analogue of octacosanol. Lipids 2009;44(10); 907-916.

https://doi.org/10.1007/s11745-009-3338-y PMid:19763655

[12]. Horiuchi N \& Maeda T. Statins and bone metabolism. Oral Disease 2006;12: 85-101.

https://doi.org/10.1111/j.1601-0825.2005.01172.x PMid:16476028

[13]. Zhao J, Yue W, Zhu MJ, Sreejayan N \& Du M. AMPactivated protein kinase (AMPK) cross-talks with canonical Wnt signaling via phosphorylation of beta-catenin at Ser 552. Biochemical and Biophysical Research Communications 2010;395:146-151. https://doi.org/10.1016/j.bbrc.2010.03.161 PMid:20361929 PMCid:PMC2864303

[14]. Sun-Ju Yi, Hyerim Lee, Jisu Lee, Kyubin Lee, Junil Kim, Yeojin Kim, Jae-Il Park, and Kyunghwan Kim. Bone Remodeling: Histone Modifications as Fate Determinants of Bone Cell Differentiation. Int J Mol Sci. 2019;20(13): 3147.

https://doi.org/10.3390/ijms20133147

PMid:31252653 PMCid:PMC6651527

[15]. Dike N. Kalu. The Rat as an Animal Model of Postmenopausal Bone Loss. In book: Sex Steroids and Bone 1994;167-181. https://doi.org/10.1007/978-3-662-03043-1_9

[16]. Han S, Hagan DL, Taylor JR, Xin L, Meng W, Biller SA, Wetterau JR, Washburn WN, Whaley JM. Dapagliflozin, a selective SGLT2 inhibitor, improves glucose homeostasis in normal and diabetic rats. Diabetes. 2008;57(6):1723-9. https://doi.org/ 10.2337/db07-1472 PMid:18356408

[17]. Parhizkar S, Ibrahim R, Abdul Latiff L. Incision choice in laparatomy: a comparison of two incision techniques in ovariectomy of rats. World Appl Sci J. 2008; 4(4): 537-40.

Int J Anat Res 2020, 8(3.3):7728-37. ISSN 2321-4287
[18].Doube M, K3 osowski MM, Arganda-Carreras I, Cordelières FP, Dougherty RP, Jackson JS, et al. Free and extensible bone image analysis in Image J. Bone 2010; 47(6):1076-9.

https://doi.org/10.1016/j.bone.2010.08.023 PMid:20817052 PMCid:PMC3193171

[19]. Bancroft JD, Gamble M (2008) Theory and practice of histological techniques. 6th ed. Elsevier, Philadelphia: Churchill Livingstone.121-134.

[20]. Annemarie K.L, Elizabeth A.L, YuliyaKlymenko, YueyingLiu, OlegKim, MarwaAsem, KevinMcAbee , Matthew J.Ravosa, M. SharonStack. Methods for the visualization and analysis of extracellular matrix protein structure and degradation. Methods in Cell Biology 2018;143:79-95

https://doi.org/10.1016/bs.mcb.2017.08.005 PMid:29310793 PMCid:PMC6317720

[21]. Milovanovic P, Djonic D, Marshall RP, Hahn M, Nikolic S, Zivkovic V. Micro-structural basis for particular vulnerability of the superolateral neck trabecular bone in the postmenopausal women with hip fractures. Bone 2012;50 (1):63-68.

https://doi.org/10.1016/j.bone.2011.09.044 PMid:21964412

[22]. Thompson DD, Simmons HA, Pirie CM, Ke HZ. FDA Guidelines and animal models for osteoporosis. Bone 1995;17 (4 Suppl): 125S-133S. https://doi.org/10.1016/8756-3282(95)00285-L

[23]. An YH; Freidman RJ (1998) Animal Models in Orthopaedic Research. Boca Raton: CRC Press. p. 284.

[24]. Meng W, Ellsworth BA, Nirschl AA et al. Discovery of dapagliflozin: a potent, selective renal sodium-dependent glucose cotransporter 2 (SGLT2) inhibitor for the treatment of type 2 diabetes. J Med Chem 2008;51:1145-1149. https://doi.org/10.1021/ jm701272q PMid:18260618

[25]. Ferrannini E, Ramos SJ, Salsali A, Tang W, List JF. Dapagliflozin monotherapy in type 2 diabetic patients with inadequate glycemic control by diet and exercise: a randomized, double-blind, placebocontrolled, phase 3 trial. Diabetes Care 2010; 33: 17-24. https://doi.org/10.2337/dc10-0612 PMid:20566676 PMCid:PMC2945163

[26].Bilal Osta, Giulia Benedetti, and Pierre Miossec. Classical and paradoxical effects of TNF-á on bone homeostasis. Frontiers in Immunology 2014; 5(48):1-9. https://doi.org/10.3389/fimmu.2014.00048

[27]. Yumei Ye, Xiaoming Jia, Mandeep Bajaj,Yochai Birnbaum. Dapagliflozin Attenuates $\mathrm{Na}+\mathrm{H}+\mathrm{Ex}-$ changer-1 in Cardiofibroblasts via AMPK Activation. Cardiovascular Drugs and Therapy 2018;32(6):553-558.

https://doi.org/10.1007/s10557-018-6837-3 PMid:30367338

[28]. A.Solini, G.Sebastiani, L.Nigi, E.Santini, C.Rossi, F.Dotta. Dapagliflozin modulates glucagon secretion in an SGLT2-independent manner in murine alpha cells. Diabetes \& Metabolism 2017;43(6): 512-520. https://doi.org/10.1016/ j.diabet.2017.04.002 PMid:28499695 
[29]. Chang YK, Choi H, Jeong JY, Na KR, Lee KW, Lim BJ, Choi DE. Dapagliflozin, SGLT2 Inhibitor, Attenuates Renal Ischemia-Reperfusion Injury. PLoS One 2016; 11(7):e0158810.

https://doi.org/10.1371/journal.pone.0158810 PMid:27391020 PMCid:PMC4938401

[30]. Yao D1, Wang S, Wang M, Lu W. Renoprotection of dapagliflozin in human renal proximal tubular cells via the inhibition of the high mobility group box 1 receptor for advanced glycation end products nuclear factor êB signaling pathway. Mol Med Rep. 2018; 18(4):3625-3630.

https://doi.org/10.3892/mmr.2018.9393

[31]. Naoto Terami, Daisuke Ogawa, Hiromi Tachibana, Takashi Hatanaka, Jun Wada, Atsuko Nakatsuka, Jun Eguchi, Chikage Sato Horiguchi, Naoko Nishii, Hiroshi Yamada, Kohji Takei, and Hirofumi Makino. Long-Term Treatment with the Sodium Glucose Cotransporter 2 Inhibitor, Dapagliflozin, Ameliorates Glucose Homeostasis and Diabetic Nephropathy in db/db Mice. PLoS One 2014;9(6): e100777. https://doi.org/10.1371/journal.pone.0100777 PMid:24960177 PMCid:PMC4069074

[32]. Ali F, Abdel-Wahabab, Ghazi A.Bamagousa, Randa M.Al-Harizy, Naser A.ElSawy, Naiyer Shahzad, Ibrahim A.Ibrahim, Saeed S. AlGhamdi. Renal protective effect of SGLT2 inhibitor dapagliflozin alone and in combination with irbesartan in a rat model of diabetic nephropathy. Biomedicine \& Pharmacotherapy 2018;103:59-66.

https://doi.org/10.1016/j.biopha.2018.03.176 PMid:29635129

[33]. Ying-Ying Chen, Tsung-Tien Wu, Chiu-Yi Ho, TungChen Yeh, Gwo-Ching Sun, Ya-Hsin Kung, Tzyy-Yue Wong, Ching-Jiunn Tseng, and Pei-Wen Cheng. Dapagliflozin Prevents NOX- and SGLT2-Dependent Oxidative Stress in Lens Cells Exposed to FructoseInduced Diabetes Mellitus. Int J Mol Sci. 2019;20(18): 4357.

https://doi.org/10.3390/ijms20184357

PMid:31491943 PMCid:PMC6770809

[34].Bolinder J, Ljunggren Ö, Johansson L, et al. Dapagliflozin maintains glycaemic control while reducing weight and body fat mass over 2 years in patients with type 2 diabetes mellitus inadequately controlled on metformin. Diabetes Obes Metab. 2014;16(2):159-69. https://doi.org/10.1111/ dom.12189 PMid:23906445

[35].Ljunggren Ö, Bolinder J, Johansson L, et al. Dapagliflozin has no effect on markers of bone formation and resorption or bone mineral density in patients with inadequately controlled type 2 diabetes mellitus on metformin. Diabetes Obes Metab. 2012;14(11):990-9.

https://doi.org/10.1111/j.1463-1326.2012.01630.x PMid:22651373
[36]. Nauck MA, Del Prato S, Meier JJ et al. Dapagliflozin versus glipizide as add-on therapy in patients with type 2 diabetes who have inadequate glycemic control with metformin: a randomized, 52-week, double-blind, active-controlled noninferiority trial. Diabetes Care 2011; 34: 2015-2022.

https://doi.org/10.2337/dc11-0606

PMid:21816980 PMCid:PMC3161265

[37]. List JF, Woo V, Morales E, Tang W, Fiedorek FT. Sodium-glucose cotransport inhibition with dapagliflozin in type 2 diabetes. Diabetes Care 2009;32:650-657.

https://doi.org/10.2337/dc08-1863

PMid:19114612 PMCid:PMC2660449

[38]. De Jong MA, Petrykiv SI, Laverman GD, et al. Effects of dapagliflozin on circulating markers of phosphate homeostasis. Clin J Am Soc Nephrol. 2019;14:66-73.

https://doi.org/10.2215/CJN.04530418

PMid:30559106 PMCid:PMC6364523

[39]. Hafiza A. Sharaf, Nermeen M. Shaffi , Fatma A. Morsy, Manal A. Badawi, Naglaa F. Abbas. Role of some phytoestrogens in recovering bone loss: histological results from experimental ovariectomized rat models. Journal of the Arab Society for Medical Research 2015;10:65-75. https://doi.org/10.4103/1687-4293.175880

[40]. Smith R, Wordsworth P. Osteoporosis. Clinical and Biochemical disorders of the skeleton 2005;123.

[41]. Ji-Yu Wang, Yan-Zhen Cheng, Shuang-Li Yang, Min An, Hua Zhang, Hong Chen and Li Yang. Dapagliflozin Attenuates Hyperglycemia Related Osteoporosis in ZDF Rats by Alleviating Hypercalciuria. Frontiers in Endocrinology 2019;10:1-14.

https://doi.org/10.3389/fendo.2019.00700 PMid:31781028 PMCid:PMC6856656

[42]. Kohan D, Fioretto P, Tang W. Long-term study of patients with type 2 diabetes and moderate renal impairment shows that dapagliflozin reduces weight and blood pressure but does not improve glycemic control. Kidney Int. 2014;4:962-971. https://doi.org/10.1038/ki.2013.356 PMid:24067431 PMCid:PMC3973038

How to cite this article: Amany Ali Nawar, Nancy Mohamed El Sekily. PROMISING EFFECT OF DAPAGLIFLOZIN ON OVARIECTOMIZED RAT MODEL OF OSTEOPOROSIS. Int J Anat Res 2020;8(3.3):7728-7037. DOI: 10.16965/ijar.2020.204 